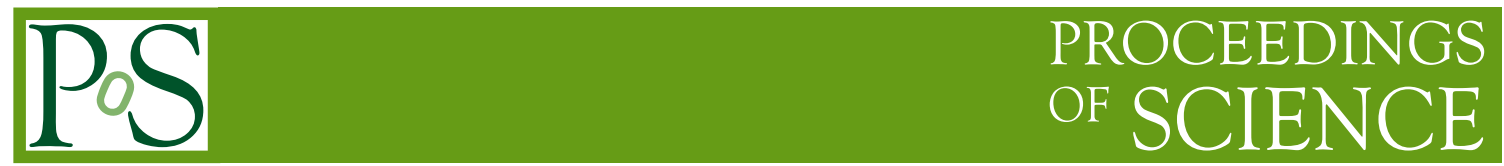

\title{
Experimental review of sterile neutrino searches
}

\section{DAVID LHUILLIER*CEA Saclay}

E-mail: david.lhuillierecea.fr

The publication of the reactor antineutrino anomaly has revived the search for a sterile neutrino state with $\Delta m^{2}$ in the $1 \mathrm{eV}^{2}$ range. A large experimental program is under development to search for a new oscillation pattern in the disapearance of $v_{e}, \bar{v}_{e}$ at short distance from compact neutrino generators. After a short status of the reactor anomaly we describe the proposed experiments with emphasis on the complementary detection technologies.

The European Physical Society Conference on High Energy Physics 18-24 July, 2013

Stockholm, Sweden

${ }^{*}$ Speaker. 


\section{The reactor antineutrino anomaly and the $4^{\text {th }}$ neutrino hypothesis}

In the 80 's the total $\beta$ spectra associated with the fission of uranium and plutonium isotopes have been accurately measured $[1,2]$. The associated electron antineutrino $\left(\bar{v}_{e}\right)$ spectra were obtained via a conversion procedure with a remarkable 5\% relative uncertainty across the relevant energy range. These reference spectra have feed the field of neutrino oscillation searches at reactors for the next 30 years. Early 2011 the prediction of the $\bar{v}_{e}$ spectra emitted by nuclear reactors has been re-evaluated [3,4]. After correcting for some approximations in the convertion procedure it was shown that the emitted flux should be increased by about $4 \%$. This triggered a re-analysis of all published neutrino measurements revealing a significant deficit of observed $\bar{v}_{e}$ rate with respect to the prediction [5]. Including the results of the recent $\mathrm{km}$ baseline experiments the latest update of the mean deficit is $6.4 \%$ with a $2.7 \sigma$ significance [8].

This so-called reactor anomaly is strengthened by independent disappearance of $v_{e}$ 's from intense $\beta$ sources observed by the Gallex [6] and Sage [7] experiments. The combined significance, above $3 \sigma$ level, makes it difficult to explain these anomalies by a single systematic effect. Another interpretation is the existence of a new neutrino state, a sterile neutrino, that doesn't interact with matter but does mix with the 3 known states at short distance. If the new mass eigenstate is sufficiently high above the others the oscillation pattern would be rapidly smeared out by the experimental resolution effects, leaving the observed imprint of a mean deficit after few $10 \mathrm{~m}$ of propagation. Constaining the new oscillation parameters with the combined data, the contours of figure 1 are obtained.

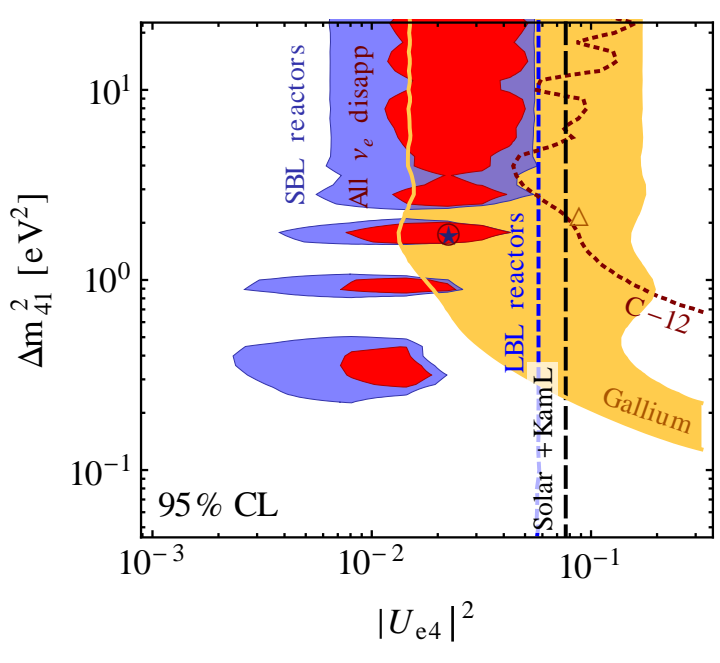

Figure 1: Allowed regions at 95\% CL for the new mixing parameters taken from [9]. The red shaded region is the combined region from $v_{e}$ and $\bar{v}_{e}$ disappearance data sets. The best fit is illustrated by the star.

On the $v_{\mu}\left(\bar{v}_{\mu}\right)$ side, the situation is more confused with some $v_{\mu} \rightarrow v_{e}$ appearance data in favor of a new ocsillation in the same $\Delta m^{2}$ range but $v_{\mu}$ disappearance data in clear tension with this hypothesis. Still an evidence of a sterile neutrino state would be a major discovery and a large experimental program is under development to search for it [10]. Choice is made in this review to focuss on the tests of the $v_{e}, \bar{v}_{e}$ disappearance anomalies with reactor and sources experiments.

\section{Experimental specifications to test the $\bar{v}_{e}$ disappearance anomalies}

The goal of all proposed experiments is to go beyond the current deficit and observe new ocsillation patterns with sensitivity to the expected dependence of the neutrino rate on energy (E) and distance (L). The contour of figure 1 sets the relevant range of oscillation length $L_{\text {osc }} \simeq$ 
$2.47 E / \Delta m_{n e w}^{2}$ in the range of [1-10] $\mathrm{m}$. Therefore the search for sterile neutrinos requires compact (meter scale) sources and short baselines $(1-10 \mathrm{~m})$. The sought sensitivity to the mixing amplitude implies a high statistical accuracy with a sample of more than $10^{5}$ detected neutrinos and a control of the systematics at the few $\%$ level. Resolving the oscillation pattern implies

$$
\delta L<<L_{o s c}, \quad \frac{\delta E}{E}<<\frac{\delta L_{o s c}}{L}
$$

The first condition is easily met by standard vertex reconstruction or rough segmentation of the detector. The energy resolution has to be in the [10-20] \% range at $1 \mathrm{MeV}$ requiring some care of light yield and edge effects.

The detection technique of most experiments described below relies on the inverse $\beta$-decay (IBD) process where the incident $\bar{v}_{e}$ flux interacts with the hydrogen nuclei of a scintillating medium to produce a positron and a neutron in the final state: $\bar{v}_{e}+p \rightarrow e^{+}+n$. The prompt energy deposition of the positron gives access to the energy of the neutrino while the differed capture of the neutron provides a discriminant signature of the process. This IBD final state determines the possible accidental and correlated background contributions.

The prompt event of an accidental pair is typically a $\gamma$-ray from the inherent radioactivity of the detector materials or induced by the neutrino generator itself. The accidental delayed event is either a capture of a thermal neutron or a high energy external $\gamma$ mimicking the signal of a neutron capture. The accidental background is usually suppressed by passive shielding around the active volume of the detector and careful choice of materials. The remaining contribution can be measured online very accurately by opening many off-time coincidence windows for each prompt candidate. Therefore the uncertainty associated to the accidental background is dominated by the statistical fluctuations of the (signal+background) rate, with little impact of the subtraction itself or knowledge of the detector parameters.

The cosmic rays are a source of correlated background which by definition passes all time and energy cuts of the neutrino selection. The underlying mecanisms are the production of neutrons and $\beta-n$ emitters by spallation reactions. The very short baseline reactor experiments presented here are all at shallow depth and therefore quite exposed to this background.

It is mandatory that any source of correlated background induced by the neutrino generator itself (reactor core or $\beta$ source) be negligible. Indeed the remaining contribution of this background after the selection cuts could only be assessed by simulation.

\section{Reactor experiments}

Nuclear reactors are very intense sources of neutrinos allowing high detection rates with tonscale target mass, about $1700 \mathrm{v} / \mathrm{MW} /$ ton/year assuming 50\% detection efficiency. Research reactors offer very compact cores with typical dimensions of the order $1 \mathrm{~m}$ and very short baselines are available. At commercial reactors the effects of the larger core size and longer baseline is partially compensated by a higher rate. However in the search for new oscillation patterns, the vertex and energy resolution effects move the sensitivity toward smaller $\Delta m^{2}$ [11].

As already mentionned the shallow detph of reactor experiments with short baselines exposes them to a correlated background induced by the cosmic rays. The standard protection is a combination 
of an optimized overburden, an active veto to tag the nearby muons and a possible discrimination of the expected prompt and/or delayed signals (see sections 3.1 and 3.3). After all the selection cuts, the remaining cosmic background is subtracted using the reactor OFF measurements corresponding to about half of the total operating time at research reactors.

The main challenge of the short baseline experiments is the mitigation of the reactor induced

\begin{tabular}{|lr|c|cccc|}
\hline Projects & $\begin{array}{r}\text { Ref } \\
\text { technic }\end{array}$ & Detection & $\begin{array}{c}P_{\text {th }} \\
(\mathrm{MW})\end{array}$ & $\begin{array}{c}M_{\text {target }} \\
\text { (tons) }\end{array}$ & $\begin{array}{c}L \\
(\mathrm{~m})\end{array}$ & $\begin{array}{c}\text { Depth } \\
\text { (m.w.e.) }\end{array}$ \\
\hline \hline Nucifer & {$[12]$} & Gd-LS & 70 & 0.75 & 7 & 13 \\
Stereo & {$[10]$} & Gd-LS & 50 & 1.75 & {$[8.8-11.2]$} & 18 \\
Neutrino 4 & {$[13]$} & Gd-LS + mov-det & 100 & 2.2 & {$[6-12]$} & few \\
DANSS & {$[14]$} & PS + high-seg + mov-det & 3 & 0.9 & {$[9.7-12.2]$} & 50 \\
Solid & {$[15]$} & PS + high-seg + n-tag & {$[45-80]$} & 3 & {$[6-8]$} & 10 \\
Hanaro & & LS + n-tag & 30 & 0.5 & 6 & few \\
US project & {$[16]$} & Gd-LS + 2-det & $20-120$ & 1 \& 10 & $4 \& 18$ & few \\
CARR & {$[17]$} & LS or $D_{2} 0+2$-det & 60 & - & $7 \& 15$ & few \\
\hline
\end{tabular}

Table 1: Main features of proposed reactor experiments. Abbreviations of detection technics: Gd-LS, Gd-doped liquid scintillator; PS, plastic scintillator; high-seg, highly segmented detector; n-tag, enhanced discrimination of the neutron capture signal; mov-det, movable detector; 2-det, two detectors setup.

background. In the following subsections the numerous projects under development in the world are grouped into categories of detection technics which pros and cons are briefly presented. The summary table 1 lists the main features of all projects.

\subsection{Gd-Loaded liquid scintillators}

The detection of neutrinos by doped liquid scintillators is now a mature technology. High transparency and year scale stability has been demonstrated for large scale production (see for instance [18]). The high light yield favors a good energy resolution improving the sensitivity to an oscillation pattern. In case of correlated background induced by external fast neutrons an online pulse shape discrimination (PSD) is possible to reject the prompt proton recoils.

The most common dopant is gadolinium because of its very high neutron-capture cross-section. Typical capture time is about $20 \mu \mathrm{s}$, an order of magnitude smaller than in pure LS, reducing by the same factor the integration of accidental background in the time window of the IBD process. The $8 \mathrm{MeV} \gamma$-cascade subsequent to a neutron capture on Gd is way above all natural radioactivity. However this neutron tagging remains sensitivte to captures on other metals $(\mathrm{Fe}, \mathrm{Ni}, \mathrm{Al})$ that emits high energy $\gamma$ 's as well. Therefore when installed in the vicinity of a reactor core a neutrino detecor must be well protected inside thick lead and polyethylene shielding. Also the $8 \mathrm{MeV}$ cascade usually consists of 3 or $4 \gamma$ 's with a high probability of energy leakage in meter scale detectors.

Among this class of projects Nucifer [12], installed at the Osiris reactor in France and initialy designed for reactor surveillance, might be the first to bring new constraints on the reactor anomaly. After a last shielding upgrade, in progress, the best fit area of fig.1 could be within reach with the foreseen 2014 data. Two dedicated projects based on the same technology but with improved 
detector response and shielding are under development. The Stereo detector [?] will be installed next year at the ILL reactor in France. Its segmented target surrounded by an active outer crown allows to cover the anomaly contour with good sensitivity. The Neutrino4 project [13] is of similar concept with a movable detector extending its baseline range. It will be installed next year at the SM-3 reactor in Dimitrovgrad (Russia).

\subsection{Highly segmented detectors}

For the oscillation analysis, a vertex resolution of few $10 \mathrm{~cm}$ is good enough (Eq.2.1). However a finer segmentation of the detector provides valuable information on the topology of the events and helps the background rejection. The first information is the space correlation between the prompt and delayed vertices while accidentals are uniformly distributed in the detector volume. Then the topology of energy deposition of the prompt or delayed event themsleves can be exploited. For instance a prompt positron will produce a compact track concentrating all the kinetic energy followed by the 2 anihilation $\gamma$ 's, possibly detected in different detector cells. Such a pattern can be discriminable from the external $\gamma$ background. The DANSS project [14] proposes to use a stack of 2500 PS stripes assembled in crossed X,Y planes wraped in Gd-loaded reflective cover. The whole detector, to be installed underneath the $3 \mathrm{GW}$ th core of the Kalinin Nuclear Power Plant in Russia, is well protected from cosmic rays and can move vertically on a lifting plateform. A very high rate of $10^{4}$ events/day is expected with a moderate energy resolution inherent to the readout of eack plastic stripe by wavelength-shifter fibers.

\subsection{Enhanced neutron discrimination}

At close distance from a core an enhanced discrimination of the neutron capture is a way to get rid of all the high energy $\gamma$ background induced by the reactor. A good candidate to replace the gadolinium is the ${ }^{6} \mathrm{Li}$ isotope leading to a very specific final state after neutron capture: ${ }^{6} \mathrm{Li}+n \rightarrow{ }^{4}$ $H e+t$. In Li doped-LS (Hanaro project) the energy deposition of the neutron capture is very compact however the signal of the two heavy charged particles in the final state is quenched by about an order of magnitude. This shrinks the neutron capture signal below $1 \mathrm{MeV}$ where the backgroun rate is high. A new development is proposed by the Solid project [15] where the detector consists of a stack of thousands of $5 \times 5 \times 5 \mathrm{~cm}$ plastic cubes with one face coupled to a ${ }^{6} \mathrm{LiF}: \mathrm{ZnS}$ layer. After a neutron capture on ${ }^{6} \mathrm{Li}$ the helium and tritium nuclei sparkle inside the $\mathrm{ZnS}$ layer producing a very discriminant signal, well separated from all electromagnetic backgrounds. This unique combination of very high segmentation and enhanced neutron tagging has a high potential of background rejection. However as for the DANSS project the readout of each plastic cube is made by a net of fibers with limited light output and energy resolution. Also the rejection of cosmic rays induced background requires further study. The validation of this detection technic is in progress with a prototype currently tested at the Mol reactor in Belgium.

\subsection{Multi-baseline concept}

The concept of two short baseline detectors on a same site is proposed by a US project [16] and a chinese project at the CARR site [17]. The idea is to install a $1 \mathrm{~m}^{3}$ scale detector at very short distance $(5 \mathrm{~m}$ ) and a second one of bigger volume at about $15 \mathrm{~m}$. The relative comparison 
of both detectors removes most of the normalisation systematics although the two detectors are not identical. The significantly larger range of baseline probed extends the sensitivity to the $0.1-1 \mathrm{eV}^{2}$ domain of $\Delta m^{2}$ in a complementary way to the other projects. First data could be taken in 2016.

\section{Source experiments}

Other possible generators of neutrinos providing intense fluxes and short baselines are high activity radioactive sources. The idea is to place the source at the center or in the immediate vicinity of a large LS-based neutrino detector like KamLAND or Borexino. From measurements of the source activity and spectral data of the signle source isotope, the emitted neutrino spectrum can be known with great accuracy. On the detection side the above mentionned detectors have been running for years. They have demonstrated very good resolution ( $15 \mathrm{~cm}$ on position, $5 \%$ on energy) and have a low and well controlled background. Therefore the main challenge of these experiment is the production and transportation of the source to the detector laboratory. But once it is installed, good data quality is almost guaranteed.

\subsection{Neutrino generators}

Intense generators of neutrinos have already been produced for the SAGE [7] and GALLEX [6] experiments with MCi scale activities. Sources of ${ }^{51} \mathrm{Cr}$ and ${ }^{37} \mathrm{Ar}$ were obtained by irradiation of samples of ${ }^{50} \mathrm{Cr}$ and ${ }^{40} \mathrm{Ca}$ in the thermal neutron flux of a reactor. Sub-MeV mono-energetic neutrino lines are emitted via the electron conversion process and detected in the LS by the scattering process $v_{e}+e \rightarrow v_{e}+e$. An irreductible background of this measurement is the solar $v_{e}$ neutrino flux. Other possible contribution are the radioactivity of the materials and impurties of the source itself. The SOX project [19] proposes to place a $10 \mathrm{MCi}{ }^{51} \mathrm{Cr}$ source underneath the Borexino detector at $8.25 \mathrm{~m}$ from its center. A new oscillation signal induced by a sterile neutrino would be signed by a modulation of the detected rate per unit volume as a function of the distance to the source. Data from this first phase are expected in 2015. In a second phase the deployement of a ${ }^{144} \mathrm{Ce}$ anti-neutrino source is foreseen, similar to the CeLand project described in the next section.

\subsection{Antineutrino generators}

The IBD detection process is an asset of antineutrino generators. The interaction cross-section is larger than the neutral current channels and the final products of the reaction provide a powerful rejection of the background. Combining these two advantages a comparable sensitivity can be reached with a source activity significantly lower than the neutrino generators [20]. The CeLAND project [21] proposes to produce a ${ }^{144} \mathrm{Ce}$ source of $75 \mathrm{kCi}$ and install it close to the KamLAND detector. This isotope is one of the very few candidate following a favorable $\beta$-decay chain. A first transition toward ${ }^{144} \mathrm{Pr}$ acts as a reservoir of activity with a low end-point energy below IBD threshold but a long lifetime (285 days). The search of a sterile neutrino exploits the second transition toward ${ }^{144} \mathrm{Nd}$ with a $3 \mathrm{MeV}$ end-point. The expected oscillation signal mainly develops along the available baseline range of [5-15] m. The good energy resolution of KamLAND provides some extra sensitivity to the distorsion of the energy spectrum above the IBD threshold. The source can be produced in the Mayak facility (russia) by reprocessing spent fuel of nuclear reactors. Transportation issues are being worked out for a data taking in 2015 . 


\section{Conclusion}

A complete experimental program is under development to search for a new disappearance pattern of electronic neutrinos at short distance from compact neutrino generators. All experiments have a nice discovery potential with a sensitivity at $5 \sigma$ level or above for the best fit of the new oscillation parameters. While liquid or plastic scintillators are common to all projects a number of different technologies are proposed to tag the neutrino signal and to reject the background. The combination of reactors and sources experiments offers complementary ranges of neutrino energy and baselines for a good characterization of the possible new oscillation parameters. Most projects expect their first data in 2015-16. Within 5 years from now the relevant area of the contour plot of figure 1 should be covered with high confidence level and that the question of the existence of sterile neutrinos linked to the reactor anomaly be settled.

\section{References}

[1] F. von Feilitzsch, A. A. Hahn and K. Schreckenbach, Phys. Lett. 118B, 162 (1982).

[2] A. A. Hahn et al., Phys. Lett. 218B, 365 (1989).

[3] T. Mueller et al., Phys. Rev. C83, 054615 (2011), [hep-ex/1101.2663].

[4] P. Huber, Phys. Rev. C84, 024617 (2011).

[5] G. Mention et al., Phys. Rev. D83, 073006 (2011).

[6] W. Hampel et al., Phys. Lett. B447, 127-133 (1999).

[7] J. N. Abdurashitov et al., Phys. Rev. C60, 055801 (1999).

[8] G. Mention et al., to be published.

[9] J. Kopp et al., JHEP 1305, (2013) 050.

[10] K. N. Abazajian et al., [arXiv: hep-ph/1204.5379].

[11] K. M. Heeger et al., Phys. Rev. D87, 073008 (2013).

[12] A. S. Cucoanes et al., J. Phys. Conf. Ser. 375, 042063 (2012), M. Vivier et al., AAP2013 conference, [https: / / indico. cern. ch/contributionDisplay . py? session Id $=0$ \& contribId $=6$ \& $\operatorname{confId}=245969]$.

[13] A. P. Serebrov et al., [arXiv:hep-ph/1205.2955]; [arXiv:hep-ph/1310.5521].

[14] I. Alekseev et al., [arXiv:hep-ph/1305.3350]; M. Danilov, [these proceedings].

[15] A. Vacheret et al., AAP2013 conference, [https://indico.cern. ch/contributionDisplay.py?session Id=0\&contribId=12 \& $\operatorname{confId}=245969]$.

[16] Z. Djurcic et al., [arXiv:hep-ph/1309.7647].

[17] G. Guo et al., [arXiv:hep-ph/1303.0607].

[18] C. Aberle et al., JINST 7 (2012) P06008.

[19] G. Bellini et al., JHEP 1308 (2013) 038.

[20] M. Cribier et al., Phys. Rev. Lett. 107 (2011) 201801.

[21] A. Gando et al., [arXiv: hep-ph/1312.0896]. 